\title{
The ISN Oby25 Global Snapshot Study
}

\author{
John Feehally \\ International Society of Nephrology, Brussels, Belgium
}

\author{
Key Words \\ Acute kidney injury - International Society of Nephrology · \\ 0by25 - Dehydration - Dialysis - Low- and middle-income \\ countries
}

\begin{abstract}
The International Society of Nephrology's (ISN) 0by25 initiative aims to prevent avoidable deaths from acute kidney injury (AKI) by 2025, most of which occur in low and lower middle-income countries (LLMICs). To increase evidence about the epidemiology of AKI, especially in LLMICs, ISN conducted a 'Global Snapshot', a multinational, cross-sectional study in which 322 physicians from 72 countries in 6 continents identified 3,664 adults and 354 children with AKI who were under their care of which $45 \%$ were from LLMICs, nevertheless lowincome countries were under-represented. In LLMICs, patients with $\mathrm{AKI}$ were younger, and community acquired $\mathrm{AKI}$ was more common. Hypotension (40\%) and dehydration (39\%) were the most common causes of AKI. Dehydration was a more common cause in LLMIC, as were sepsis, pregnancy-related $\mathrm{AKI}$ and animal envenomation. Acute dialysis was performed in $23 \%$ of patients. Eight percent had a clinical indication for this but were not dialyzed. In LLMICs, lack of resources (16\%) and inability to afford therapy (30\%) accounted for almost half of these cases. Overall mortality at 7 days was $11 \%$ and was higher in LLMICs. Complete recovery from AKI occurred in $30 \%$ of patients and partial recovery $37 \%$, and was more often complete in LLMICs. The 0 by 25 Global Snapshot provides new information about the world-
\end{abstract}

\begin{tabular}{ll}
\hline KARGER & $\begin{array}{l}\text { ( } 2016 \text { The Author(s) } \\
\text { Published by S. Karger AG, Basel } \\
\text { 0250-6807/16/0686-0029 } \$ 39.50 / 0\end{array}$ \\
E-Mail karger@karger.com & This article is licensed under the Creative Commons Attribution- \\
www.karger.com/anm & $\begin{array}{l}\text { NonCommercial-NoDerivatives 4.0 International License (CC BY- } \\
\text { NC-ND) (http://www.karger.com/Services/OpenAccessLicense). } \\
\text { Usage and distribution for commercial purposes as well as any dis- } \\
\text { tribution of modified material requires written permission. }\end{array}$
\end{tabular}

\section{Introduction} countries (LMICs) [2]. would be amenable in intervention to reduce preventable deaths.

(c) 2016 The Author(s)

Published by S. Karger AG, Basel

Acute kidney injury (AKI) is the term used to describe sudden loss of kidney function. Although there are multiple causes of AKI, dehydration is an important primary cause often associated with hypotension. Dehydration also is an important contributory influence when other etiologic factors exist. AKI often can be reversed, in the early stages, with simple measures such as fluid replacement. Even if oliguric AKI is not prevented, recovery of renal function after 1-2 weeks is common, assuming dialysis treatment is available to replace renal function until recovery occurs.

The 0by 25 AKI initiative of the International Society of Nephrology (ISN) aims to prevent all avoidable deaths from AKI across the world by 2025 [1]. The great majority of avoidable deaths occur in low- and middle-income

In high-income countries (HICs), progress in AKI epidemiology (especially in the critically ill population), has translated into better prevention, diagnosis and treatment of AKI, although the standard of care is not always achieved [3]. However, in low-income countries (LICs) and LMICs, the epidemiology of AKI is poorly described because the limited availability of human resources and 
health infrastructure are associated with poor recognition and treatment of AKI. In these countries, the few available studies suggest that a considerable proportion of AKI cases and their adverse clinical consequences could be prevented or attenuated [1].

Progress toward the ambitious goal of 0by 25 is based on 3 elements, all emphasizing AKI in LMIC. First, increasing the evidence base about AKI; second, improving awareness about AKI through advocacy; third, developing interventions that will improve detection and care of AKI in low resource settings.

\section{Methods}

To improve the information available about AKI across the globe in 2014, ISN conducted a 'Global Snapshot', a multinational, cross-sectional study involving physicians recruited by an open invitation of the ISN and partnering nephrology and critical care societies.

Physicians chose an 'index day' during the period October 2014 to December 2014 and identified potential cases of AKI among patients under their care who were first identified within 3 days of the index day. Baseline serum creatinine (SCr) was defined as the lowest SCr value during the previous 12 months. If no baseline SCr was available, the reference SCr was defined as the first SCr at patient evaluation. We applied modified Kidney Disease: Improving Global Outcomes (KDIGO) AKI criteria [4] on the index day and 7 days later to identify which of these potential cases were confirmed as AKI. We included AKI cases due to any etiology, in adults and children, whether from hospital or non-hospital settings. Outcomes included all cause patient mortality, need for dialysis and complete or partial renal recovery. History and laboratory studies at the time of study enrollment determined if there was underlying chronic kidney disease. Patients were considered to have either de novo AKI, AKI-on-CKD or AKI with unknown prior kidney history. AKI stages (KDIGO criteria) were determined at diagnosis, dialysis start, at 7 days and last observation. Countries were classified into income groups on the basis of gross national income per person, using thresholds defined by the World Bank Atlas method [5].

\section{Results}

Three hundred twenty-two physicians from 72 countries across the world participated in the Global Snapshot. This included cases from North America, Latin America and the Caribbean, Western Europe, Eastern Europe, Middle East, South Asia, East and Southeast Asia, Oceania and Africa; i.e., total of 3,664 adults and 354 children with AKI. Most cases (45\%) were from low and lower middleincome countries (LLMICs) followed by upper middleincome countries (UMICs) (29\%) and HIC (26\%). We combined the data from LLMICs because we only had 195 patients from LIC, of which more than $75 \%$ were from 2 countries. Median age was 60 years, but was younger in LLMICs (median age 50 years). Chronic heart and liver diseases were more frequent in patients from HIC and UMIC, whereas pre-existing CKD was less prevalent in LLMICs. The majority had developed community acquired AKI (60\%), but this was higher in LLMIC (80\%).

Hypotension (40\%) and dehydration (39\%) were the most common causes of AKI. Dehydration was more common in LLMICs (46\%). Most of the 'dehydration' episodes were associated with inadequate oral intake (60\%), followed by vomiting (44\%). Sepsis, pregnancy-related $\mathrm{AKI}$ and animal envenomation were also more common in LLMIC. Approximately $75 \%$ of patients received fluids as the initial AKI treatment. Crystalloid was the most common intravenous fluid utilized. Use of albumin and starch was employed more often in UMICs. Dialysis was performed in $23 \%$ of patients, and it was more commonly started at a higher level of SCr in LLMICs. Of the nondialyzed patients, $8 \%$ had a clinical indication for renal replacement therapy but were not dialyzed, and this occurred with equal frequency across income categories. In LLMICs, lack of resources (16\%) and inability to afford therapy (30\%) accounted for almost half of these cases. Conversely, in HICs, the main reason to withhold dialysis was a perception of 'futility' (79\%). Mortality at 7 days was $11 \%$ and was significantly higher in dialyzed (17\%) versus non-dialyzed (9\%) patients. In LLMICs, mortality was significantly higher in cases of community acquired AKI, in those requiring critical care facilities and in children.

Complete recovery from AKI occurred in $30 \%$ of patients and partial recovery in $37 \%$. AKI recovery was more often complete in LLMICs regardless of whether dialysis was performed during the hospitalization or not. The results of the Global Snapshot have been published in detail elsewhere [6].

\section{Discussion}

The 0by25 Global Snapshot is the first worldwide, prospective cross-sectional study of AKI designed to assess similarities and differences in the recognition and management of AKI across 6 continents. We developed this study to provide baseline evidence for the ISN 0by25 initiative which targets preventable deaths from AKI [1], and focuses on identifying elements that would be amenable to intervention, to reduce preventable deaths.
30

Ann Nutr Metab 2016;68(suppl 2):29-31 DOI: $10.1159 / 000446202$
Feehally 
Although dehydration, infections and sepsis were important etiological factors in all countries (and contributed to hypotension and shock as the most common precipitants for AKI), they probably reflected different exposures and patient factors across different study sites. The higher incidence of cardiac failure, postoperative AKI and nephrotoxic agents in the older HIC and UMIC patients may reflect exposure to multiple diagnostic (e.g., contrast imaging) and therapeutic interventions in hospitals. In contrast, in the younger LLMIC patients, the drivers of AKI were dehydration, infections, animal envenomation and complicated pregnancy [7].

Access to dialysis in LLMICs is often limited, with variations seen even in different regions of the same country or the same city. Unsurprisingly, we found that the lack of resources was a frequent reason for not providing dialysis in LLMICs. Peritoneal dialysis was infrequently utilized across all countries, despite its advantages in low resource settings, as is being demonstrated recently by the ISN Saving Young Lives Project, which has successfully developed and implemented programs for teaching and providing peritoneal dialysis in several LLMICs [8], which may help to reduce AKI-related mortality and morbidity in these areas.

The observed high AKI mortality is concerning, particularly in LLMIC where almost $80 \%$ of the patients had community acquired AKI, which reportedly is associated with better outcomes [9]. Appropriate management of patients with incomplete kidney recovery may delay the progressive loss of kidney function [10] and improve their later quality of life. Worldwide educational campaigns for a full range of healthcare personnel should emphasize not only the importance of early detection and prompt management of AKI, but also the need for longterm follow-up of AKI patients.

The Global Snapshot is a unique prospective cross-sectional study designed to capture regional differences in recognition, management and outcomes of AKI, but nevertheless it has significant limitations. It represents a convenience sample of the incidence of AKI, and the summary data are unlikely to be representative of any single country. The paucity of data from LIC and outpatient settings highlights the challenges in obtaining information from low resource sites. In addition, most of the LLMIC centers were located in referral hospitals in large cities, and thus cases of AKI in rural settings and small urban centers are under-represented in this study. It is probable that dehydrating gastroenteritis is an even more common cause of AKI in very low resource community settings, but this study cannot confirm this assumption. Seasonal variations in AKI also were not captured in the limited time duration for this study, which identified cases between October and December 2014. Finally, a lack of trained personnel, infrastructure issues (e.g., intermittent electricity and internet connections), a heavy workload and time constraints are recognized factors limiting participation in studies, and inevitably favored the collection of data from better-resourced settings within LLMIC [2]. Our findings thus represent a limited view of the true burden of AKI in outpatient settings and in LICs and emphasize the need for new strategies to close these knowledge gaps.

\section{Declaration of Interests}

J. Feehally received an honorarium from UCB Pharma in 2015, but has no other interests to declare.

\section{References}

1 Mehta RL, Cerdá J, Burdmann EA, et al: International society of nephrology's 0by 25 initiative for acute kidney injury (zero preventable deaths by 2025): a human rights case for nephrology. Lancet 2015;385:2616-2643.

2 Lewington AJ, Cerdá J, Mehta RL: Raising awareness of acute kidney injury: a global perspective of a silent killer. Kidney Int 2013;84: 457-467.

3 Adding Insult to Injury. A Review of the Care of Patients Who Died in Hospital with a Primary Diagnosis of Acute Kidney Injury (Acute Renal Failure). A Report by the National Confidential Enquiry into Patient Outcome and Death, 2009.
4 Kidney Disease: Improving Global Outcomes (KDIGO) Acute Kidney Injury Work Group: KDIGO clinical practice guideline for acute kidney injury. Kidney Int Suppl 2012;2:1138.

5 Bank W: GNI per capita, Atlas method (current US\$). 2015. http://dataworldbankorg/indicator/NYGNPCAPCD.

6 Mehta RL, Burdmann EA, Cerdá J, et al: Current practice for recognition and management of acute kidney injury: the ISN 0by25 Global Snapshot study. Lancet 2016, in press.

7 Jha V, Parameswaran S: Community-acquired acute kidney injury in tropical countries. Nat Rev Nephrol 2013;9:278-290.
8 Smoyer WE, Finkelstein FO, McCulloch MI, Carter M, Brusselmans A, Feehally J: 'Saving Young Lives' with acute kidney injury: the challenge of acute dialysis in low-resource settings. Kidney Int 2016;89:254-256.

9 Wonnacott A, Meran S, Amphlett B, Talabani B, Phillips A: Epidemiology and outcomes in community-acquired versus hospital-acquired AKI. Clin J Am Soc Nephrol 2014;9:1007-1014

10 Cerda J, Liu KD, Cruz DN, Jaber BL, Koyner JL, Heung M, Okusa MD, Faubel S; AKI Advisory Group of the American Society of $\mathrm{Ne}$ phrology: Promoting kidney function recovery in patients with AKI requiring RRT. Clin J Am Soc Nephrol 2015;10:1859-1867. 